\title{
Admitting an elderly patient with solid tumor in the intensive care unit: what do we have to look for?
}

\author{
Carmen Silvia Valente Barbas ${ }^{1,2}$, Ellen Pierre de Oliveira $^{1}$, João Valente Barbas Filho ${ }^{1}$ \\ ${ }^{1}$ Divisão de Pneumologia, Instituto do Coração, Hospital das Clinicas HCFMUSP, Faculdade de Medicina, Universidade de São Paulo, São Paulo, \\ SP, Brazil; ${ }^{2}$ UTI-Adultos, Hospital Israelita Albert Einstein, São Paulo, SP, Brazil \\ Correspondence to: Carmen Silvia Valente Barbas. Associate Professor of Pneumology and Intensive Care Medicine of the University of São Paulo, \\ São Paulo, SP, Brazil; Assistant Physician Adult ICU Albert Einstein Hospital, Av. Dr Eneas de Carvalho Aguiar 455, 6 Andar-Respiratory ICU, São \\ Paulo, SP, Brazil. Email: carmen.barbas@gmail.com. \\ Provenance: This is an invited Editorial commissioned by Section Editor Dr. Xue-Zhong Xing [National Cancer Center (NCC)/Cancer Hospital, \\ Chinese Academy of Medical Sciences (CAMS) and Peking Union Medical College (PUMC), Beijing, China]. \\ Comment on: Auclin E, Charles-Nelson A, Abbar B, et al. Outcomes in elderly patients admitted to the intensive care unit with solid tumors. Ann \\ Intensive Care 2017;7:26.
}

Submitted Aug 16, 2017. Accepted for publication Sep 21, 2017.

doi: 10.21037/jtd.2017.09.114

View this article at: http://dx.doi.org/10.21037/jtd.2017.09.114

Admitting an elderly critically ill patient with solid tumor in the intensive care unit (ICU) for treatment was always a matter of debate among intensive care practitioners because the doubts about the impact that the critical therapy could have in the prognosis of the cancer patients concerning the survival rate and moreover, in the quality of life after the critical event. The patient with cancer can be discharged from the ICU but be weakened and unable to continue the cancer treatment or have more difficulties in the everyday life leaving many doctors and family members opt for palliative care.

Recently, remarkable advances in medical technology regarding early and better cancer diagnosis and new options of cancer treatment as well as the advances in critical care health technology and humanization results in the possibility of a personalized ICU intensive treatment that can give the elderly cancer patients a much better multidisciplinary support.

In order to assess patient outcomes and identify factors associated with 90-day mortality and antineoplastic treatment resumption in elderly patients with metastatic and non-metastatic solid cancers admitted to the ICU, Auclin and colleagues reported in the Annals of Intensive Care (1) a retrospective analysis of 262 patients from 2,327 eligible elderly patients with solid tumors (14.3\%), admitted to the ICU of the European Georges Pompidou Hospital (Paris,
France) between 2009 and 2014. Their elderly cancer patients were admitted because of sepsis $(30.5 \%)$, acute respiratory failure (28.2\%), and neurologic disorders (8.0\%). Their patients were very ill (SAPS 2 of $61.9 \pm 22.5$ ), $60.3 \%$ had metastatic disease, $51.5 \%$ needed invasive ventilatory support, $48.1 \%$ needed inotropic drugs and $12.1 \%$ needed renal replacement therapy. When comparing the ICU mortality in elderly patients with solid tumors to the elderly critically ill patients without solid tumors the authors didn`t find statistical differences ( $33.6 \%$ vs. $32.6 \%, \mathrm{P}=0.75)$. The 90-day mortality was $51.9 \%$. A multivariate analysis showed that a higher SAPS 2 score (HR 1.05; 95\% CI: 1.03-1.06, $\mathrm{P}<0.0001)$ and the primary site of the cancer being the head and neck $(\mathrm{P}=0.01)$ were significantly associated with 90 -day death in elderly patients with cancer admitted to the ICU, but not the presence of metastatic disease or the patient anti-neoplastic previous treatments. Cancer treatment was resumed after ICU stay in more than half of their patients.

Most of previous studies that analyzed prognosis of patients with cancer admitted to ICUs revealed that the survival rates depend more of the severity of the critical illness than the presence of the cancer or patient age (2-6), fact that was confirmed by the Auclin cohort too.

After analyzing the data that studied the outcomes of elderly patients with solid tumors admitted to the ICU we came to the conclusion: let's talk to the cancer patients 
and their families about treating early and in the ICU the acutely ill cancer patients. Let's do our best to give them the best prognosis and quality of life as possible!

\section{Acknowledgements}

None.

\section{Footnote}

Conflicts of Interest: The authors have no conflicts of interest to declare.

\section{References}

1. Auclin E, Charles-Nelson A, Abbar B, et al. Outcomes in elderly patients admitted to the intensive care unit with solid tumors. Ann Intensive Care 2017;7:26.

2. Bonomi MR, Smith CB, Mhango G, et al. Outcomes

Cite this article as: Barbas CS, de Oliveira EP, Barbas Filho JV. Admitting an elderly patient with solid tumor in the intensive care unit: what do we have to look for? J Thorac Dis 2017;9(11):4141-4142. doi: 10.21037/jtd.2017.09.114 of elderly patients with stage IIB-V non-small cell lung cancer admitted to the intensive care unit. Lung Cancer 2012;77:600-4.

3. Soares M, Toffart AC, Timsit JF, et al. Intensive care in patients with lung cancer: a multinational study. Ann Oncol 2014;25:1829-35.

4. Soares M, Caruso P, Silva E, et al. Characteristics and outcomes of patients with cancer requiring admission to intensive care units: a prospective multicenter study. Crit Care Med 2010;38:9-15.

5. Azevedo LCP, Caruso P, Silva UVA, et al. Outcomes for patients with câncer admitted to the ICU requiring ventilatory support: results from a prospective multicenter study. Chest 2014;146:257-66.

6. Sprung CL, Sakr Y, Vincent JL, et al. An evaluation of systemic inflammatory response syndrome signs in the Sepsis Occurrence In Acutely Ill Patients (SOAP) study. Intensive Care Med 2006;32:421-7. 